Urologe 2010 · 49:597

DOI 10.1007/s00120-010-2250-7

Online publiziert: 21. April 2010

c) Springer-Verlag 2010
F.M.E. Wagenlehner $\cdot$ W. Weidner

Klinik für Urologie, Kinderurologie und Andrologie,

Justus-Liebig-Universität Gießen, Gießen

\title{
Urogenitale Infektionen und deren Folgen
}

Infektionen im Bereich des Urogenitaltraktes zählen zu den häufigsten Infektionen überhaupt. Sie stellen auch und insbesondere in der Urologie ein signifikantes klinisches Problem dar. Teilweise unterschätzte Langzeitfolgen sind damit vergesellschaftet. Die zunehmende Antibiotikaunempfindlichkeit der Erreger ist ein für alle Urologen belastendes Problem.

Uropathogene Erreger sind hochspezialisierte und selektionierte Bakterien, die eine Reihe, auf das spezifische Immunsystem des Urogenitaltraktes ausgerichtete Pathogenitätsfaktoren aufweisen und somit akute oder chronische Infektion und Entzündung im Urogenitaltrakt auslösen. Der Urogenitaltrakt selbst weist hochspezialisierte und teilweise einzigartige immunologische Strukturen auf, um die kontinuierliche Auseinandersetzung mit den pathogenen Erregern ohne signifikante Begleitschäden führen zu können.

Vor diesem Hintergrund sollen in diesem Themenheft neben basiswissenschaftlichen Erläuterungen zu uropathogenen Bakterien und der Immunantwort sowohl die unmittelbaren als auch Langzeitfolgen von urogenitalen Infektionen besprochen werden. Dabei sind die schweren akuten Infektionen im Bereich des Harntraktes, die nicht selten in systemischen Infektionen bis hin zur Urosepsis münden können, ein besonderes Thema. Wir haben mit den Möglichkeiten der minimal invasiven, endoskopischen urologischen Therapie die Prognose der Urosepsis im Vergleich zu anderen Sepsisentitäten signifikant gebessert. Eine wichtige
Ursache stellt auch die Urolithiasis dar. Da bestimmte Formen der Harnweginfektion als Infektstein auf diese zurückzuführen sind und chronische wiederkehrende Infektionen bedingen können, bedürfen diese einer besonderen Kenntnis. Die „häufigen“ antibiotischen Therapien stellen dann eine wichtige Ursache der Antibiotikaresistenz dar, da der Antibiotikaselektionsdruck permanent gesteigert wird.

Grundsätzlich ist die Entstehung einer Antibiotikaresistenz als Langzeitfolge des Antibiotikaeinsatzes zu verstehen und ist, wie bereits oben ausgeführt, ein zunehmendes herausforderndes klinisches Problem in der Urologie, da neue effektive Antibiotikasubstanzen insbesondere im urologisch wichtigen Gramnegativen Bereich auch in absehbarer Zeit nicht zur Verfügung stehen werden. Eine antiinfektiöse Therapie mit Antibiotika hat daher im Gegensatz zu allen anderen Therapien von Erkrankungen nicht nur Auswirkung auf den individuellen Fall, sondern auch auf das gesamte medizinische Umfeld. Dieser besonderen Verantwortung muss sich jeder Arzt, der Antibiotika verschreibt, bewusst sein.

Besondere Aspekte stellen chronische Infektionen und Entzündungen des Urogenitaltraktes bei der Infertilität und die Assoziation von chronischen Entzündungen/ Infektionen mit Tumoren dar. Die Rolle der chronischen Infektion bei der Infertilität ist bisher nicht eindeutig geklärt, trotzdem finden wir bei bis zu $20 \%$ aller infertilen Patienten urogenitale Infektionen oder Entzündungen in der Anamnese. Auch im Bereich des häufigs- ten malignen Tumors des Mannes, dem Prostatakarzinom, verdichten sich die Hinweise, dass zumindestens bei der Initiierung des neoplastischen Prozesses eine chronische Entzündung eine wichtige Rolle spielen könnte.

Das Themenheft soll Ihnen darstellen, dass die Folgen der so häufigen urogenitalen Infektionen mannigfaltig sind und grundsätzliche Fragen aufwerfen. Aus diesem Grund sehen wir die Notwendigkeit, dass sich der Urologe mit diesem „alltäglichen" Problem beschäftigt, um auch vorausschauend für die Zukunft mit der besonderen Problematik der urogenitalen Infektionen und Entzündungen und ihrer Wechselwirkung umgehen zu können.

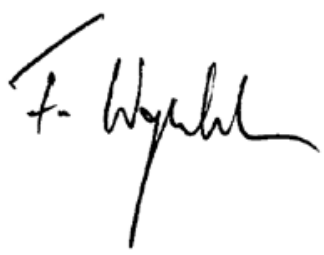

F.M.E. Wagenlehner

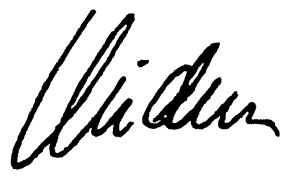

W. Weidner

\section{Korrespondenzadresse \\ PD Dr. F.M.E. Wagenlehner}

Klinik für Urologie, Kinderurologie und Andrologie, Justus-Liebig-Universität Gießen, Rudolf-Buchheim-Straße 7, 35385 Gießen

Florian.Wagenlehner@chiru.med.uni-giessen.de 